\title{
Micropatterned K-carrageenan-PVP-PEG hydrogels as a templet for head and neck cancer spheroid culture
}

\author{
Nadia Halib ${ }^{1 *}$, Zainah Adam², Maznah Mahmud ${ }^{3}$ \\ ${ }^{1}$ Department of Basic Sciences and Oral Biology, Faculty of Dentistry, Universiti Sains Islam Malaysia, Kuala Lumpur, Malaysia. \\ ${ }^{2}$ Pharmaceutical Technology Unit, Medical Technology Division, Malaysian Nuclear Agency, Kajang, Malaysia. \\ ${ }^{3}$ Biopolymer Group, Radiation Processing Technology Division, Malaysian Nuclear Agency, Kajang, Malaysia.
}

\begin{tabular}{l}
\hline ARTICLE INFO \\
\hline Received on: 13/11/2020 \\
Accepted on: 20/03/2021 \\
Available online: 05/05/2021 \\
\\
\hline Key words: \\
Hydrogels, $\kappa$-carrageenan, \\
PVP, PEG, 3D cell culture, \\
HTB43.
\end{tabular}

\begin{abstract}
Hydrogels have been demonstrated to exhibit distinct efficacy as matrices for 3D cell culture. As this research field advances, the needs for matrices combining both the benefits of natural and synthetic polymer hydrogels are becoming more apparent. In this study, kappa-carrageenan-PVP-PEG (КCA-PVP-PEG) hydrogels have been micropatterned with microwells to enhance human squamous carcinoma (HTB43 ${ }^{\mathrm{TM}}$ ) spheroid growth. It was found that the hydrogels' swelling ratios (SR) were 11.91, 11.67, and 11.67 for day 2, day 4, and day 8, respectively. A stable SR indicated no further swelling of hydrogels in the cell culture media, hence allowing spheroid growth in an intact mold without the rupture or dissolution of microwell templet. The comparison between unirradiated and irradiated hydrogel Fourier transform infrared (FTIR) spectra showed a slight shift of O-H (stretch) from 3,327 to 3,382 $\mathrm{cm}^{-1}$ and $\mathrm{CH}$ and $\mathrm{CH}_{2}$ (stretch) from 2,919 to 2,921 cm respectively, whereas other functional peaks remained unchanged, suggesting the formation of interpenetrating network as the primary mechanism for hydrogels formation. The morphological study revealed the spherical shape of spheroids with measurements between 130 and $220 \mu \mathrm{m}$. Spheroids emit a signal in 4',6-diamidino-2-phenylindole (DAPI) and fluorescein isothiocyanate (FIT-C) channels indicating spheroid viability. In conclusion, micropattern $\kappa$ CA-PVP-PEG hydrogels deemed suitable as a templet for generation and $\mathrm{HTB} 43^{\mathrm{TM}}$ spheroid growth for $3 \mathrm{D}$ cell culture application.
\end{abstract}

\section{INTRODUCTION}

Conventional cell culture models are in 2D mode or monolayer where cells are grown on a flat surface, adhere and spread on the surface, forming unnatural cell attachments, and hence render $2 \mathrm{D}$ cell cultures inadequate in mimicking the living system, thus making them unreliable tools to be used in in vivo drug efficacy and toxicity studies. In addressing these issues, researchers have now look forward to 3D cell cultures, an artificially created environment in which biological cells are permitted to grow or interact with their surroundings in all three dimensions (Andersen et al., 2015). This arrangement will allow the cultures to provide a model that more accurately resembles the living system.

"Corresponding Author

Nadia Halib, Department of Basic Sciences \& Oral Biology, Faculty of Dentistry, Universiti Sains Islam Malaysia, Kuala Lumpur, Malaysia. E-mail:nadia.halib@usim.edu.my
In this light, hydrogels have been deemed to be a suitable scaffold for $3 \mathrm{D}$ cell cultures as they have the ability to mimic the natural extracellular matrix (ECM). Hydrogels possess tunable mechanical strength, topography, porosity, and biocompatibility, allowing nutrient and oxygen perfusion that enhance and promote cell growth (Tibbitt and Anseth, 2009). Moreover, the fabrication process of hydrogels can be combined with micropattern technologies including soft lithography, micromolding, and photolithography that enable the precise control of microscale features including surface topography (Gauvin et al., 2012). Among these, micromolding is a feasible technique that can be used to fabricate micropattern on thermoplastics, elastomers, and hydrogels in producing specific geometries and sizes (Cui et al., 2017) where precursor polymers are initially molded and subsequently gelled with the addition of gelling agents such as divalent cations (Khademhosseini and Langer, 2007).

Microwell is one of the examples of geometries pattern that can be fabricated onto hydrogels surface via micromolding technique. It has proven to be particularly suitable for controlling 
the size of spheroids while maintaining high cell viability during/ after spheroid formation due to the ECM-mimicking biophysical properties of hydrogels (Gong et al., 2015; Karp et al., 2007; Mirab et al., 2019). These microwell platforms have been prepared using a variety of polymers such as agarose (Napolitano et al., 2007), chitosan (Fukuda et al., 2006), poly(ethylene glycol) (PEG) (Karp et al., 2007), and gelatine methacrylate (Peela et al., 2016).

Carrageenan is a hydrophilic polysaccharide derived from red seaweed that closely resembles glycosaminoglycans due to the presence of a backbone that is comprised of alternate units of sulphated D-galactose and 3,6-anhydrogalactose linked through $\alpha$-1,3- and $\beta$-1,4-glycosidic linkage. There are three main classes of carrageenan, namely, kappa $(\kappa)$, iota $(l)$, and lambda $(\lambda)$, with mono, di-, and trisulphate groups, respectively (Necas and Bartosikova, 2013). Several studies have reported the biocompatibility of $\kappa$-carrageenan $(\kappa \mathrm{CA})$, thus deeming its suitability as biomedical devices. Recently, several works have also demonstrated the chondrogenic potential of $\kappa$ CA (Popa et al., 2015).

The thermoresponsive nature of $\kappa \mathrm{CA}$ allows an easy formation of hydrogels in the presence of cations (Vignesh et al., 2018). It undergoes coil-helix conformational transition, rendering ionotropic and thermotropic hydrogels that dissolve when heated (Chronakis et al., 2000). However, the ionic interactions produce strong but brittle hydrogels. Several approaches have been proposed by researchers including the introduction of photo crosslinkable methacrylate moieties on the $\kappa \mathrm{CA}$ backbone to create physically and chemically cross-linked hydrogels (Mihaila et al., 2013). In our case, we introduced polyvinylpyrrolidone (PVP) and PEG to this natural-synthetic polymer blend. PEGs are oligomers and/or polymers of ethylene oxide widely used as a lubricant in the biomedical field. The addition of PEG managed to incorporate elasticity and other properties such as adhesion and tackiness while improving hydrogel qualities (Ajji et al., 2005). Meanwhile, a previous study done by Mangione et al. (2005) indicated that more ordered and strong hydrogels were obtained in the presence of $\mathrm{K}+$ with respect to $\mathrm{Na}+$ ions. The hydrogel properties obtained using mixtures of the two cosolutes also depended on the $[\mathrm{K}+] /$ $[\mathrm{Na}+]$ ratio (Mangione et al., 2005).

As previously mentioned, $\kappa$ CA can easily form hydrogels with tunable mechanical properties, making it an excellent material for micropatterning (Michel et al., 1997; Vignesh et al., 2018). However, in a small scale and dimension such as cell culture scaffold, $\kappa C A$ hydrogels easily break, hence handling the hydrogels during the tissue culturing process rather difficult. Therefore, in this research, we introduced PVP and PEG into $\kappa C A$ to incorporate elasticity and improve its tensile properties (De Silva et al., 2011). Furthermore, PVP and PEG are water-soluble polymers which can produce a homogenous mixture with $\kappa \mathrm{CA}$. We also used micromolding technique to fabricate microwells onto kappa-carrageenan-PVP-PEG ( $\mathrm{CA}-\mathrm{PVP}-\mathrm{PEG}$ ) hydrogels as a template for spheroid growth. Hydrogels' swelling properties and Fourier transform infrared (FTIR) spectra were determined, while its biocompatibility as tissue culture templet was elucidated by seeding human squamous carcinoma cells (HTB43). The spheroid growth in the $\kappa$ CA-PVP-PEG hydrogels' microwells was morphologically assessed by measuring its size and shape whereas cell viability was determined with Hoechst 33,342 and CalceinAM staining.

\section{MATERIALS AND METHODS}

\section{Materials}

The $\kappa$ CA powder was locally produced, PVP (Mw $160 \mathrm{kDa})$ and PEG (4 kDa) were purchased from Merck, and Dulbecco's Modified Eagle Medium (DMEM), fetal bovine serum (FBS), trypsin, and antibiotic solution (penicillin-streptomycin $5,000 \mathrm{U} / \mathrm{ml}$ ) were purchased from Thermo Fisher Scientific (Waltham, MA). Hoechst 33,342, Calcein-AM, and propidium iodide were purchased from Merck (Darmstadt, Germany).

\section{Fabrication of micropatterned кCA-PVP-PEG hydrogels}

The $\kappa$ CA-PVP-PEG hydrogels were prepared by mixing 5\% $\mathrm{\kappa CA}$ (in $0.1 \mathrm{M}$ sodium acetate), 15\% PVP (in distilled water), and 2\% PEG (in distilled water) with the ratio of 9:1:1. The mixture was thoroughly stirred with a magnetic bar to form a homogenous solution. Micropatterns were imprinted onto $\kappa \mathrm{CA}$ PVP-PEG hydrogels by pouring the mixture into a silicone mold with microwells cast and left to solidify at room temperature for 30 minutes. Later, the hydrogels were sterilized under gamma irradiation at $25 \mathrm{kGy}$.

\section{Swelling property}

The swelling property of $\kappa$ CA-PVP-PEG hydrogels was determined by calculating its swelling ratio (SR) every 2 days for the period of 8 days. The hydrogels were hydrated in cell culture media and the SR was calculated as

$$
\text { Swelling ratio }(\mathrm{SR})=\frac{W_{h}}{W_{l}}
$$

where $W_{\mathrm{h}}$ is the weight of the hydrated hydrogels after immersion in cell culture media and $W_{1}$ is the weight of the lyophilized hydrogels (Chang et al., 2010).

\section{Fourier transform infrared spectroscopy (FTIR) analysis}

FTIR spectra of the $\kappa$ CA-PVP-PEG hydrogels were recorded using FTIR Spectra 2000 (Perkin Elmer) at room temperature. Lyophilized hydrogel samples were pulverized and mixed evenly with potassium bromide at a ratio of $1: 90$. Samples were prepared as $\mathrm{KBr}$ pellets and subjected to spectroscopy in the range of 4,000-1,300 $\mathrm{cm}^{-1}$.

\section{HTB43 spheroids culture}

HTB43 ${ }^{\mathrm{TM}}$ monolayer cells were cultured in DMEM supplemented with $10 \%(v / v)$ FBS and $1 \%(v / v)$ antibiotic solution at $37^{\circ} \mathrm{C}$ in a humid incubator with $5 \% \mathrm{CO}_{2}$. Confluent cells were trypsinized and harvested in a complete culture medium and counted using a cell counter (Nexcelom Bioscience, Lawrence, MA). HTB43 cells were seeded into the sterile micropatterned $\kappa \mathrm{CA}-\mathrm{PVP}-\mathrm{PEG}$ hydrogels at cell densities of $1.0 \times 10^{5}$ cells/ $\mathrm{ml}$ and incubated at $37^{\circ} \mathrm{C}$ in a humid incubator with $5 \% \mathrm{CO}_{2}$. Spheroids' formation and growth on day 1, day 5, and day 10 were visualized using an inverted microscope (Olympus, Japan) at 10× magnification and spheroids images were captured using a (chargecoupled device) CCD camera system (XCAM, Tokyo, Japan).

\section{Spheroids' size and viability}

Spheroids were harvested using pipette suction and the harvested HTB43 spheroids were placed in a sterile 96-well plate. 
Diameters of spheroids were measured using a Cell Reporter system (Molecular Devices, San Jose, CA). Spheroids were then incubated with DMEM containing Hoechst 33,342 (2 $\mu \mathrm{g} / \mathrm{ml})$, Calcein-AM (400 $\mathrm{nM})$, and propidium iodide $(2 \mu \mathrm{g} / \mathrm{ml})$ for 30 minutes in dark at room temperature. The stained spheroids were imaged using 4',6-diamidino-2-phenylindole (DAPI), fluorescein isothiocyanate (FIT-C) and tetramethylrhodamine-isothiocyanate (TRIT-C) filter sets on a High Content Screening system, ImageXpress $^{\circledR}$ Micro XL (Molecular Devices, San Jose, CA). Acquired images were analyzed using MetaXpress ${ }^{\circledR}$ Software.

\section{RESULTS AND DISCUSSION}

\section{Fabrication of micropatterned кCA-PVP-PEG hydrogels}

$\kappa \mathrm{CA}$ can easily form hydrogels in the presence of cations. In this case, $\kappa$ CA-PVP-PEG hydrogels were formed by ionic crosslinking of $\kappa \mathrm{CA}$ by $\mathrm{Na}+$, which established strong interaction between the polymeric chains (Vignesh et al., 2018). Micropatterning of the hydrogels by simple micromolding technique was enabled upon complete crosslinking within 30 minutes. It was found that the microwells on the surface of $\kappa \mathrm{CA}$ PVP-PEG were in fine and clean shape where there were no cracking or roughness observed at the edge of microwells (Fig. 1). The microwells' structures were intact for up to 10 days when soaked in culture media.

It is known that $\kappa \mathrm{CA}$ produced brittle hydrogels; hence, we incorporated water-soluble synthetic polymers, namely, PVP and PEG to $\kappa \mathrm{CA}$, to form interpenetrating network (IPN) hydrogels. The addition of PEG acts as a plasticizer that enables the incorporation of elasticity, thus improving hydrogel properties including adhesion and tackiness (Ajji et al., 2005). It was also observed that the strong interaction between the polymeric chains that formed the hydrogels after complete crosslinking caused the loss of hydrophilic interaction that exists between the molds and the hydrogels, which in turn facilitated the removal of micropatterned hydrogels from silicone molds. This occurrence provides a feasible micropatterning process of $\kappa$ CA-PVP-PEG hydrogels where hydrogels were easily removed using forceps to be washed with distilled water.

\section{Swelling properties of $\kappa$ CA-PVP-PEG hydrogels}

Lyophilized $\kappa$ CA-PVP-PEG hydrogels were immersed in cell culture media for the period of 8 days and SR were determined every 2 days. The SR of $\kappa$ CA-PVP-PEG hydrogels were slightly higher on day 2 and stabilized afterwards with values of 11.91 , 11.67, and 11.67 for day 2, day 4, and day 8, respectively (Fig. 2). During the first 24 hours of immersion, water molecules were absorbed into the hydrogels' network causing it to swell. However, some of the polymer chains were dissolved due to the strong ionic interaction of various salts in the cell culture media. This resulted in the reduction of overall mass and weight of $\kappa C A-P V P-$ PEG hydrogels, hence causing the slight decreased of SR from 11.91 (day 2) to 11.67 (day 4). Further immersion of hydrogels did not cause any more dissolution of polymer chains; thus, SR for day 8 remained and stabilized suggested that the $\kappa C A-P V P-$ PEG hydrogels remained physically intact in cell culture media. Hydrogels' physical integrity is an important property to maintain the shape and dimension of micropatterned microwells that serve as templets for spheroids growth, ensuring the formation of threedimensional cell structure.

\section{Fourier transform infrared spectroscopy (FTIR) analysis}

In our present study, $25 \mathrm{kGy}$ of gamma irradiation was used for crosslinking and sterilizing the hydrogels (Ajji et al., 2005). The FTIR spectra of pure $\kappa C A$, pure PVP, pure PEG, and

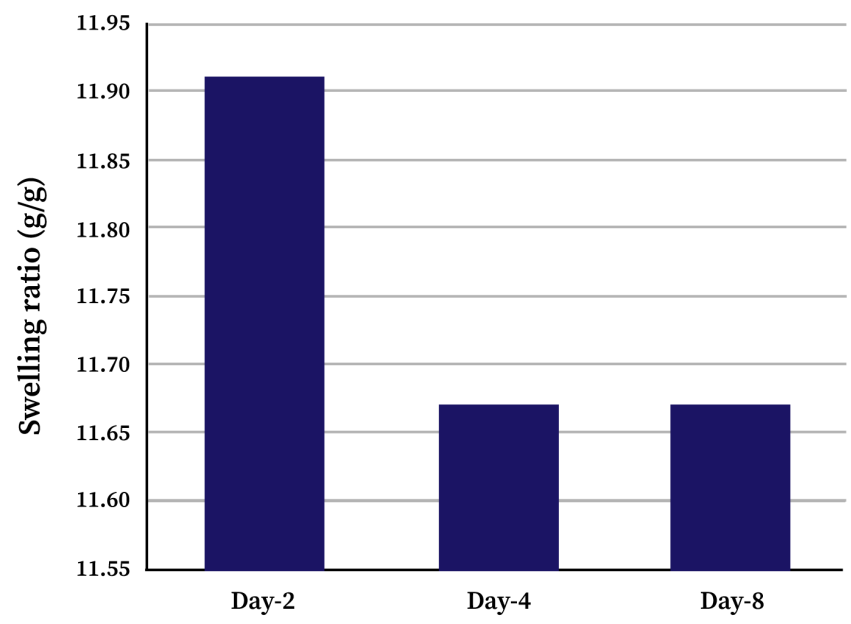

Figure 2. Swelling ratio (SR) of $\kappa$ CA-PVP-PEG hydrogels soaked in cell culture media on day 2 , day 4 , and day 8 .
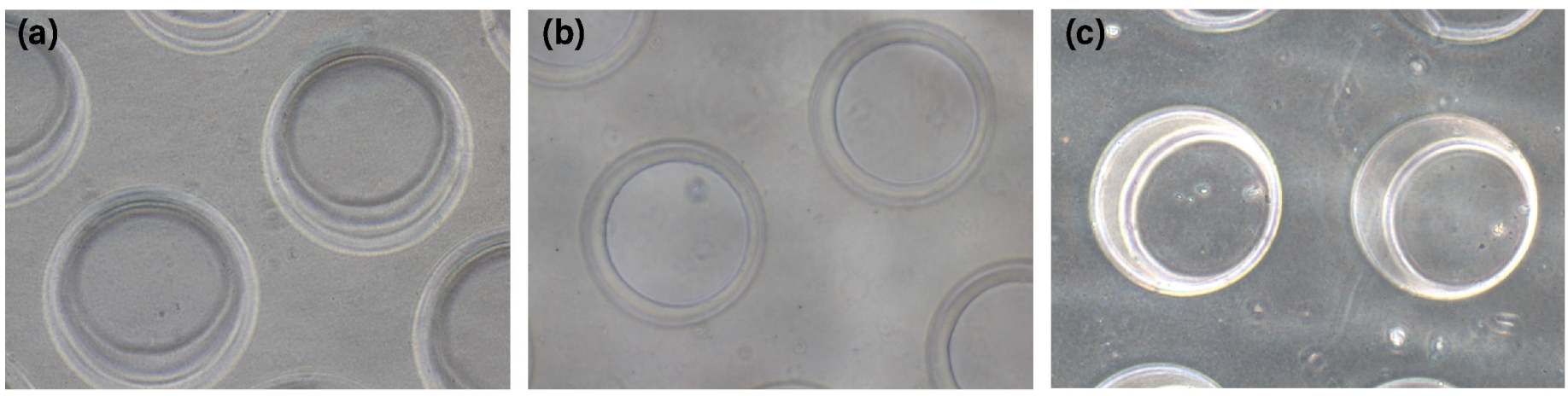

Figure 1. The morphology of microwells on the surface of $\kappa$ CA-PVP-PEG hydrogels with a diameter of approximately $200 \mu \mathrm{m}$ on (a) day 1 , (b) day 5 , and (c) day 10 in cell culture media. The images were visualized using an inverted microscope (Olympus, Japan) at 10× magnification. 
$\kappa$ CA-PVP-PEG hydrogels before and after irradiation are given in Figure 3. The major peak assignments of pure $\kappa C A$, pure PVP, and pure PEG are summarized in Table 1. The FTIR spectra of $\kappa \mathrm{CA}$ PVP-PEG hydrogels was found to consist of the functional groups from all three pure components, that is, $\mathrm{O}-\mathrm{H}$ (stretch), sulphate (stretch), glycosidic linkage, and $\mathrm{C}_{4}-\mathrm{O}-\mathrm{S}$ in galactose in pure $\kappa \mathrm{CA}$; $\mathrm{CH}_{2}$ stretch, $\mathrm{C}=\mathrm{O}$, and $\mathrm{N}-\mathrm{C}$ in pure PVP; C-O-C (stretch) in PEG (De Silva et al., 2011).

It was observed that there was no significant difference in peak pattern and peak position when the FTIR spectra of unirradiated $\kappa$ CA-PVP-PEG hydrogels was compared with the irradiated hydrogels. This finding suggested that $\kappa \mathrm{CA}$ and PEG chains were entangled within the cross-linked PVP to form an IPN. In accordance with the previous research, it is also proposed that the irradiation of $\kappa \mathrm{CA}$ with gamma rays resulted in radical formation on the $\kappa \mathrm{CA}$ macromolecule chains and these sites of

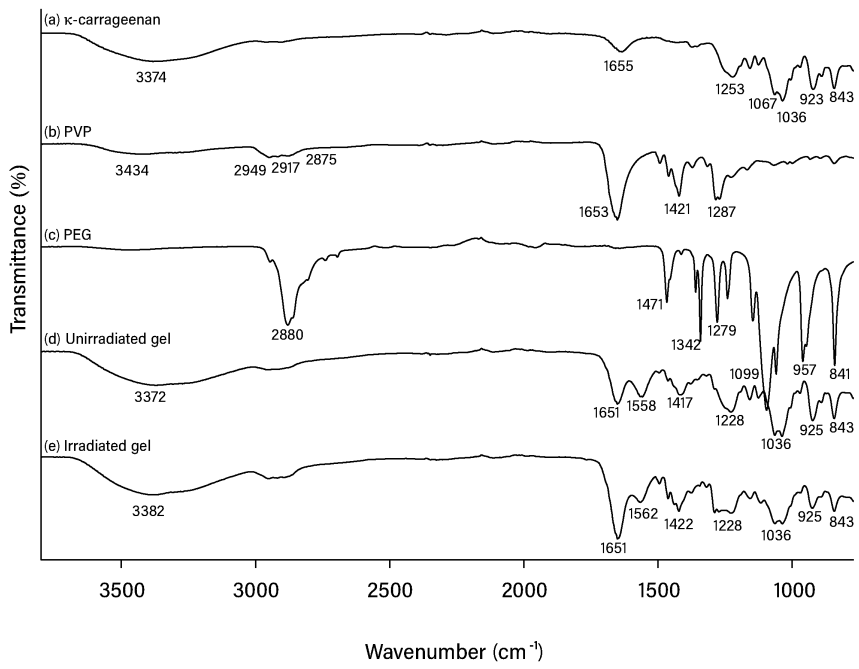

Figure 3. FTIR spectra for (a) pure $\kappa \mathrm{CA}$, (b) pure PVP, (c) pure PEG, (d)

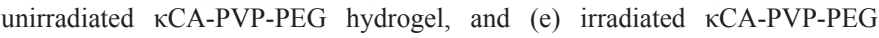
hydrogel.

Table 1. FTIR band assignments of pure $\kappa C A$, pure PVP, and pure PEG.

\begin{tabular}{|c|c|c|}
\hline Wavenumber $\left(\mathrm{cm}^{-1}\right)$ & Functional group & Reference \\
\hline \multicolumn{2}{|l|}{$\kappa \mathrm{CA}$} & \multirow{6}{*}{$\begin{array}{c}\text { (De Silva et al., } \\
\text { 2011) }\end{array}$} \\
\hline $3,400-3,000$ & O-H (stretch) & \\
\hline 1,253 & Sulphate (stretch) & \\
\hline 1,036 & Glycosidic linkage & \\
\hline 923 & C-O-C (3,6-anhydro-D-galactose) & \\
\hline 843 & $\mathrm{C}_{4}-\mathrm{O}-\mathrm{S}$ in galactose & \\
\hline \multicolumn{2}{|l|}{ PVP } & \multirow{5}{*}{$\begin{array}{c}\text { (Koczkur et al., } \\
\text { 2015) }\end{array}$} \\
\hline $2,949 / 2,917 / 2,875$ & $\mathrm{CH}$ and $\mathrm{CH}_{2}$ stretch & \\
\hline 1,653 & $\mathrm{C}=\mathrm{O}$ stretching vibration & \\
\hline 1,421 & $\mathrm{CH}$ deformation of cyclic $\mathrm{CH}_{2}$ groups & \\
\hline 1,287 & C-N vibration & \\
\hline \multicolumn{2}{|l|}{ PEG } & \multirow{4}{*}{$\begin{array}{c}\text { (Shameli et al., } \\
\text { 2012) }\end{array}$} \\
\hline 2,880 & C-H stretching & \\
\hline $1,279 / 1,099$ & $\mathrm{O}-\mathrm{H}$ and $\mathrm{C}-\mathrm{O}-\mathrm{H}$ stretching & \\
\hline $1,471 / 1,342$ & $\mathrm{C}-\mathrm{H}$ bending & \\
\hline
\end{tabular}

radical formation become the points of initiation for side chains with PVP (Abad et al., 2003). Although polysaccharide such as $\kappa \mathrm{CA}$ is known to degrade rapidly with irradiation, the unchanged FTIR spectra of hydrogels after irradiation also indicated that grafting of $\kappa$ CA to PVP or the IPN formation had diminished the degradation of $\kappa \mathrm{CA}$; in other words, PVP preserved the $\kappa \mathrm{CA}$ against gamma irradiation (Şen and Avc1, 2005).

\section{HTB43 spheroids formation}

The spheroid formation can be divided into three stages: an initial aggregation of cells, followed by spheroid compaction and finally spheroid growth (Smyrek et al., 2019). The formation of HTB43 spheroids was shown in Figure 4. It was observed that the aggregation stage of HTB43 cells has already taken place on day 1 (Fig. 4a). This is in agreement with the previous research which reported that the cell aggregation stage was found to be completed within 48 hours (Smyrek et al., 2019). On day 5 (Fig. 4b), the compaction stage of HTB43 spheroids occurred. It was observed that compact HTB43 spheroids had a spherical geometry or shape. On day 10 (Fig. 4c), compact HTB43 spheroids exhibited dark in color, probably due to the high density of cells number. However, the spheroids still look intact and viable. This suggested that micropatterned $\kappa$ CA-PVP-PEG hydrogels were compatible with HTB43 spheroids culture and had no effect on spheroids formation up to 10 days culture. The viability of HTB43 spheroids was further confirmed through fluorescent assay.

Afterward as spheroids' growth, it was observed that microwells of $\kappa$ CA-PVP-PEG hydrogels seem to be disappearing. This was probably due to the acidic condition of the culture medium ( $\mathrm{pH} 6$ ) as indicated by the changes of the culture medium's colour from red to yellow. Secretion of cellular metabolites such as lactic acid contributes to this acidic environment (Michl et al., 2019) where it might affect the anionic stability within the polymeric chains of micropatterned $\kappa$ CA-PVP-PEG hydrogels, thus creating stronger interaction between the salts as compared to interpolymeric network and hence causing the dissolution of $\kappa$ CA-PVP-PEG hydrogels after day 10. This similar finding was also reported by Mehrban et al. (2010) that study $\kappa$ CA gel cylinders seeded with $3 \mathrm{~T} 3$ cell (murine fibroblasts). The acellular samples of $\kappa \mathrm{CA}$ gel cylinders remained unchanged for the duration of the study. However, it was noticed that the addition of cells within the gel caused a gradual loss in total mass suggesting degradation of the polymer. This change was not apparent until day 10 of the study which correlates with a dramatic increase in the proliferation of encapsulated cells (Mehrban et al., 2010).

\section{Spheroids' size and viability}

Figure 5 shows images of spheroids on day 10, captured using Cell Reporter system (Molecular Devices, San Jose, CA). It is shown that spheroids formed from $\kappa$ CA-PVP-PEG hydrogels exhibited different sizes that ranged from 130 to $220 \mu \mathrm{m}$. The different sizes might be influenced by the different number of cells received by each microwell during the seeding process. Other factors that might have an effect on spheroids sizes were the amount of nutrient supplied and the time of spheroids shaking (Shi et al., 2018).

Images of HTB43 spheroids stained with a mix of three dyes, namely, Hoechst 33342, Calcein-AM, and propidium iodide, 

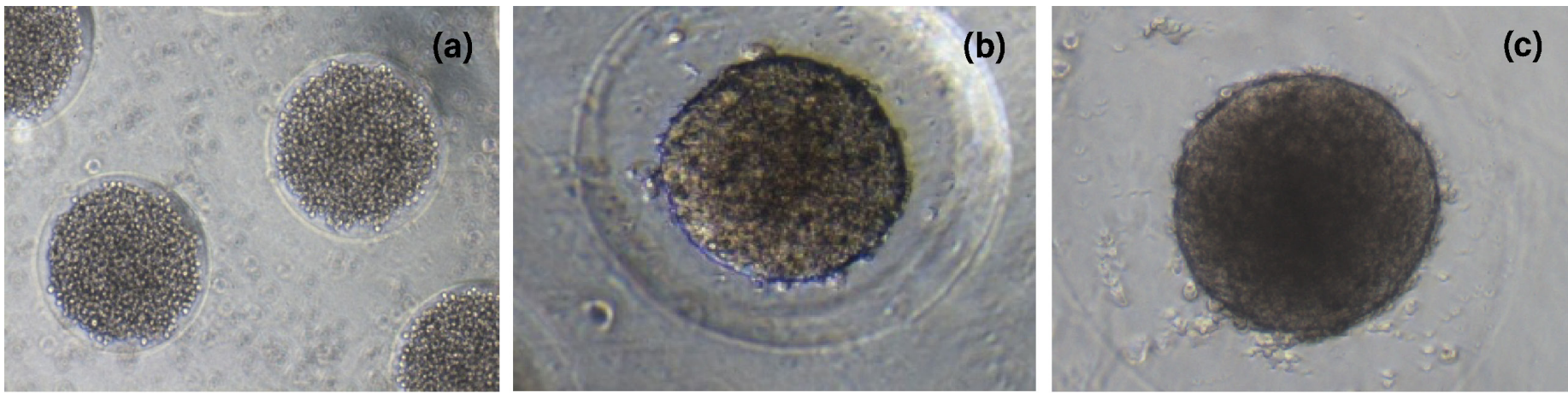

Figure 4. HTB43 spheroid formation on (a) day 1, (b) day 5, and (c) day 10.
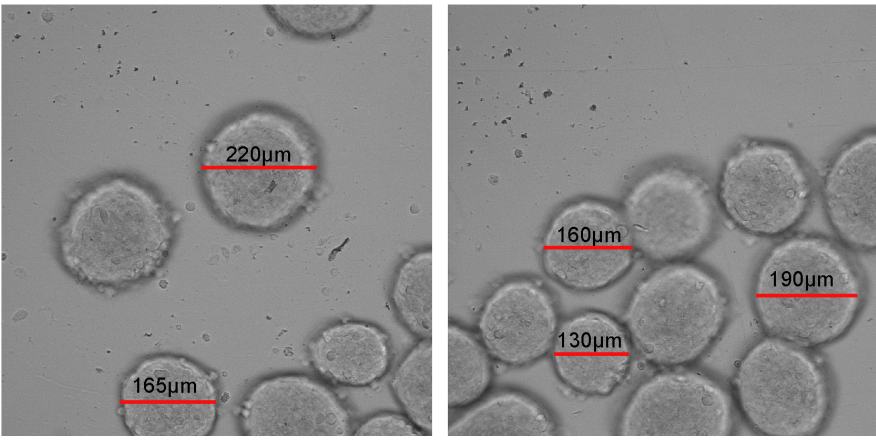

Figure 5. Harvested HTB43 spheroid on day 10 with size range between 130 $\mu \mathrm{m}$ and $220 \mu \mathrm{m}$.
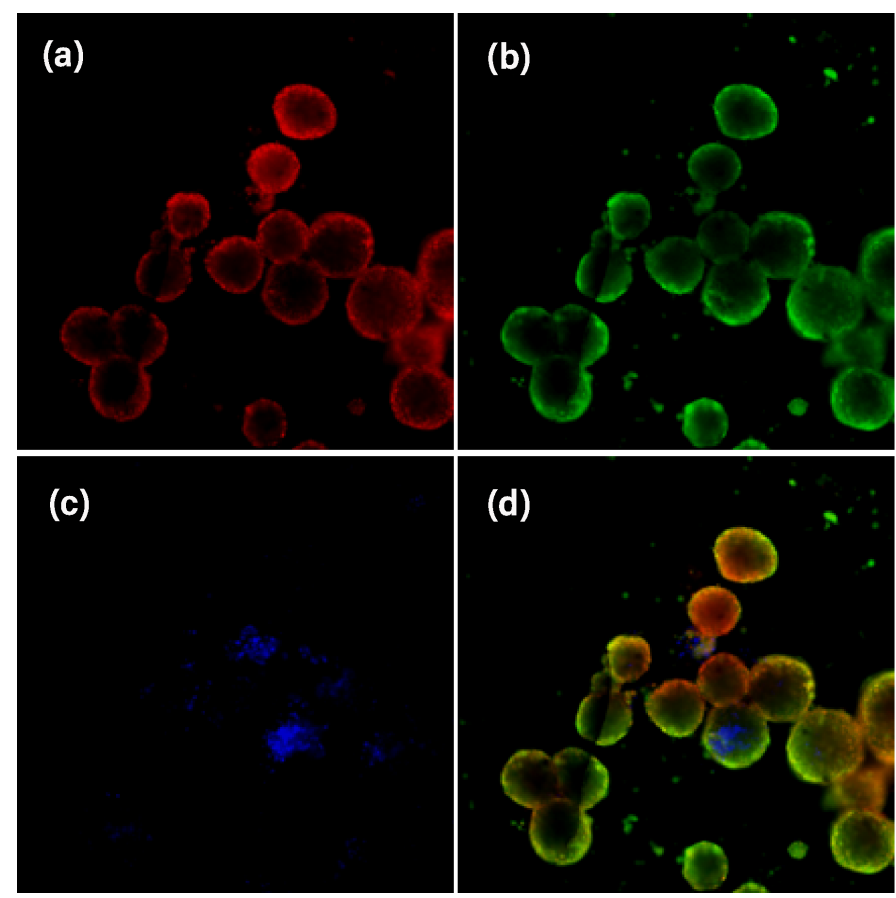

Figure 6. Representative images of HTB43 spheroids stained with a combination of three dyes: Hoechst 33342, Calcein-AM, and propidium iodide. Images were taken using DAPI (a), FIT-C (b), and TRIT-C (c) channels, respectively. A composite image of all three channels is shown at the bottom right (d). Images were acquired using an automatic imaging system (ImageXpress Micro XL) with a 20 Plan Apo $(\mathrm{NA}=0.45)$ objective. are shown in Figure $6(\mathrm{a}-\mathrm{d})$. Hoechst 33342 dye was used to detect the nucleus of single cells, Calcein-AM was used to determine metabolically active cells or live cells, and propidium iodide dye which selectively penetrates cells with damaged outer membranes was used to measure dead or necrotic cells (Sirenko et al., 2015). Images from the DAPI channel (6-a) showed the presence of the nucleus of all cells in the HTB43 spheroids. FIT-C channel images (6-b) indicated that the spheroids are still alive and viable. TRIT-C channel images (6-c) showed that there were dead or necrotic cells in HTB43 spheroids. Overlay or composite images of the three channels (6-d) showed the location of dead or necrotic cells. These findings suggested that many of the dead or necrotic cells were located at the core site of the spheroids. These were possibly due to the lack of nutrients and oxygen supplied at the spheroids'core.

A previous study reported that spheroids' structure consists of three layers: the outer proliferating layer, inner quiescent zone, and core necrotic site (Nath and Devi, 2016). At the outer layer, cells actively proliferated due to high exposure to nutrients, growth factors, and oxygen from the medium. Meanwhile, cells at the middle layer and core site received less amount of nutrients, growth factors and oxygen causing cells at the middle layer to become inactive while the cells at the core site to become necrotic (Edmondson et al., 2014). Accordingly, our cells displayed the same nature as mentioned above, thus suggesting that our $\kappa \mathrm{CA}$ PVP-PEG hydrogels served the purpose of being the potential scaffold for $3 \mathrm{D}$ cell culture.

\section{CONCLUSION}

We found that the fabrication of microwells as surface topology onto $\kappa$ CA-PVP-PEG hydrogels via a simple micromoulding technique was feasible. The microwells were in well-defined and clean shape. It was also easy to remove $\kappa \mathrm{CA}$ PVP-PEG hydrogels from the mould since complete crosslinking resulted in the loss of hydrophilic interaction between the molds and hydrogels. When seeded with HTB43 cells, кCA-PVP-PEG hydrogels were found to be stable up to day 10, providing ample time for spheroids formation. The spheroid sizes were in the range of $130-220 \mu \mathrm{m}$. The overlay images under fluorescence microscopy indicated the three layers of spheroids structure where dead or necrotic cells were located at the core site of the spheroids. These common features of spheroids suggested that our micropattern $\kappa$ CA-PVP-PEG hydrogels were a potential scaffold for $3 \mathrm{D}$ cell culture application. 


\section{ACKNOWLEDGMENTS}

The authors would like to acknowledge the assistance provided by staff in the Malaysian Nuclear Agency and financial support from both institutions (University Sains Islam Malaysia and Malaysian Nuclear Agency).

\section{AUTHOR CONTRIBUTIONS}

All authors made substantial contributions to conception and design, acquisition of data, or analysis and interpretation of data; took part in drafting the article or revising it critically for important intellectual content; agreed to submit to the current journal; gave final approval of the version to be published; and agree to be accountable for all aspects of the work. All the authors are eligible to be an author as per the international committee of medical journal editors (ICMJE) requirements/guidelines.

\section{CONFLICTS OF INTEREST}

The authors report no financial or any other conflicts of interest in this work.

\section{ETHICAL APPROVALS}

This study does not involve experiments on animals or human subjects.

\section{PUBLISHER'S NOTE}

This journal remains neutral with regard to jurisdictional claims in published institutional affiliation.

\section{REFERENCES}

Abad LV, Relleve LS, Aranilla CT, Rosa AMD. Properties of radiation synthesized PVP-kappa carrageenan hydrogel blends. Radiat. Phys Chem, 2003; 68(5):901-8.

Ajji Z, Othman I, Rosiak JM. Production of hydrogel wound dressings using gamma radiation. Nucl Instrum Methods B, 2005; 229(34):375-80.

Andersen T, Auk-Emblem P, Dornish M. 3D cell culture in alginate hydrogels. Microarrays, 2015; 4(2):133-61.

Chang C, Duan B, Cai J, Zhang L. Superabsorbent hydrogels based on cellulose for smart swelling and controllable delivery. Eur Polym J, 2010; 46(1):92-100.

Chronakis IS, Doublier JL, Piculell L. Viscoelastic properties for kappa-and iota-carrageenan in aqueous $\mathrm{NaI}$ from the liquid-like to the solidlike behaviour. Int J Biol Macromol, 2000; 28(1):1-14.

Cui X, Hartanto Y, Zhang H. Advances in multicellular spheroids formation. J R Soc Interface, 2017; 14(127):20160877.

De Silva DA, Hettiarachchi BU, Nayanajith LDC, Milani MDY, Motha JTS. Development of a PVP/kappa-carrageenan/PEG hydrogel dressing for wound healing applications in Sri Lanka. J Natl Sci Found, 2011; 39(1):25-33.

Edmondson R, Broglie JJ, Adcock AF, Yang L. Threedimensional cell culture systems and their applications in drug discovery and cell-based biosensors. Assay Drug Dev Technol, 2014; 12(4):207-18.

Fukuda J, Khademhosseini A, Yeo Y, Yang X, Yeh J, Eng G, Blumling J, Wang CF, Kohane DS, Langer R. Micromolding of photocrosslinkable chitosan hydrogel for spheroid microarray and cocultures. Biomaterials, 2006; 27(30):5259-67.

Gauvin R, Parenteau-Bareil R, Dokmeci MR, Merryman WD, Khademhosseini A. Hydrogels and microtechnologies for engineering the cellular microenvironment. Wiley Interdiscip Rev Nanomed Nanobiotechnol, 2012; 4(3):235-46.
Gong X, Lin C, Cheng J, Su J, Zhao H, Liu T, Wen X, Zhao P. Generation of multicellular tumor spheroids with microwell-based agarose scaffolds for drug testing. PloS One, 2015; 10(6):e0130348.

Karp JM, Yeh J, Eng G, Fukuda J, Blumling J, Suh KY, Cheng J, Mahdavi A, Borenstein J, Langer R. Controlling size, shape and homogeneity of embryoid bodies using poly (ethylene glycol) microwells. Lab Chip, 2007; 7(6):786-94.

Khademhosseini A, Langer R. Microengineered hydrogels for tissue engineering. Biomaterials, 2007; 28(34):5087-92.

Koczkur KM, Mourdikoudis S, Polavarapu L, Skrabalak SE. Polyvinylpyrrolidone (PVP) in nanoparticle synthesis. Dalton Trans, 2015; 44(41):17883-905.

Mangione MR, Giacomazza D, Bulone D, Martorana V, Cavallaro G, San Biagio PL. K+ and Na+ effects on the gelation properties of א-carrageenan. Biophys Chem, 2005; 113(2):129-35.

Mehrban N, Hunt N, Smith AM, Grover LM. A comparative study of iota carrageenan, kappa carrageenan and alginate as tissue engineering scaffolds. Gums and Stabilisers for the Food Industry, 2010; 15:407-13.

Michel AS, Mestdagh MM, Axelos MAV. Physico-chemical properties of carrageenan gels in presence of various cations. Int J Biol Macromol, 1997; 21(1-2):195-200.

Michl J, Park KC \& Swietach P. Evidence-based guidelines for controlling $\mathrm{pH}$ in mammalian live-cell culture systems. Commun Biol, 2019; 2(1): 1-12.

Mihaila SM, Gaharwar AK, Reis RL, Marques AP, Gomes ME, Khademhosseini A. Photocrosslinkable kappa-carrageenan hydrogels for tissue engineering applications. Adv Healthc Mater, 2013; 2(6):895-907.

Mirab F, Kang YJ, Majd S. Preparation and characterization of size-controlled glioma spheroids using agarose hydrogel microwells. PloS One, 2019; 14(1):e0211078.

Napolitano AP, Dean DM, Man AJ, Youssef J, Ho DN, Rago AP, Lech MP, Morgan JR. Scaffold-free three-dimensional cell culture utilizing micromolded nonadhesive hydrogels. Biotechniques, 2007; 43(4):494-500.

Nath S, Devi GR. Three-dimensional culture systems in cancer research: focus on tumor spheroid model. Pharmacol Ther, 2016; 163:94-108

Necas J \& Bartosikova L. Carrageenan: a review. Vet Med (Praha), 2013; 58(4): $187-205$.

Peela N, Sam FS, Christenson W, Truong D, Watson AW, Mouneimne G, Ros R, Nikkhah M. A three dimensional micropatterned tumor model for breast cancer cell migration studies. Biomaterials, 2016; 81:72-83.

Popa EG, Caridade SG, Mano JF, Reis RL, Gomes ME. Chondrogenic potential of injectable $\kappa$-carrageenan hydrogel with encapsulated adipose stem cells for cartilage tissue-engineering applications. J Tissue Eng Regen Med, 2015; 9(5):550-63.

Şen M, Avc1 EN. Radiation synthesis of poly (N-vinyl-2pyrrolidone)- $\kappa$-carrageenan hydrogels and their use in wound dressing applications. I. Preliminary laboratory tests. J Biomed Mater Res A, 2005; 74(2):187-96.

Shameli K, Bin Ahmad M, Jazayeri SD, Sedaghat S, Shabanzadeh P, Jahangirian H, Mahdavi M, Abdollahi Y. Synthesis and characterization of polyethylene glycol mediated silver nanoparticles by the green method. Int J Mol Sci, 2012; 13(6):6639-50.

Shi W, Kwon J, Huang Y, Tan J, Uhl CG, He R, Zhou C, Liu Y. Facile tumor spheroids formation in large quantity with controllable size and high uniformity. Sci Rep, 2018; 8(1):1-9.

Sirenko O, Mitlo T, Hesley J, Luke S, Owens W, Cromwell EF. High-content assays for characterizing the viability and morphology of 3D cancer spheroid cultures. Assay Drug Dev Technol, 2015; 13(7):402-14.

Smyrek I, Mathew B, Fischer SC, Lissek SM, Becker S, Stelzer EHK. E-cadherin, actin, microtubules and FAK dominate different spheroid formation phases and important elements of tissue integrity. Biol Open, 2019; 8(1):bio037051. 
Tibbitt MW, Anseth KS. Hydrogels as extracellular matrix mimics for 3D cell culture. Biotechnol Bioeng, 2009; 103(4):655-63.

Vignesh S, Gopalakrishnan A, Poorna MR, Nair SV, Jayakumar R, Mony U. Fabrication of micropatterned alginate-gelatin and k-carrageenan hydrogels of defined shapes using simple wax mold method as a platform for stem cell/induced pluripotent stem cells (iPSC) culture. Int J Biol Macromol, 2018; 112:737-44.
How to cite this article:

Halib N, Adam Z, Mahmud M. Micropatterned א-carrageenanPVP-PEG hydrogels as a templet for head and neck cancer spheroid culture. J Appl Pharm Sci, 2021; 11(05):015-021. 